Pacific Journal of 


\section{ALGEBRAS FORMED BY THE ZORN VECTOR MATRIX}

\section{TAE-IL SUH}

In the Zorn vector matrix algebra the three dimensional vector algebra is replaced by a finite dimensional Lie algebra $L$ over a field of characteristic not 2 equipped with an associative symmetric bilinear form $(a, b)$ and having the property: $[a[b c]]=(a, c) b-(a, b) c, a, b, c \in L$. We determine all the alternative algebras $\mathfrak{A}$ obtained in this way: If the bilinear form $(a, b)$ on $L$ is nondegenerate then $\mathfrak{U}$ is the split Cayley algebra or a quaternion algebra. For a degenerate form $(a, b)$, $\mathfrak{U}$ is a direct sum of its radical and a subalgebra which is either a quaternion or two dimensional separable algebra. As an immediate consequence of the first result we have shown that if the bilinear form on the Lie algebra $L$ is nondegenerate then $L$ is simple with dimension three or one.

Let $\Phi$ be a field of characteristic not two throughout this paper. Let $A$ be an anti-commutative algebra over $\Phi$ with a symmetric bilinear form $(a, b)$ which is associative, i.e., $(a c, b)=(a, c b), a, b, c \in A$, and we consider the set $\mathfrak{A}$ of $2 \times 2$ vector matrices of the form:

$$
\left(\begin{array}{ll}
\alpha & a \\
b & \beta
\end{array}\right), \alpha, \beta \in \Phi ; a, b \in A \text {. }
$$

$\mathfrak{U}$ is a vector space $\Phi$ under the usual addition, + , and multiplication by scalars. A multiplication in $\mathfrak{A}([5]$ and [2]) is defined to be:

$$
\left(\begin{array}{ll}
\alpha & a \\
b & \beta
\end{array}\right)\left(\begin{array}{ll}
\gamma & c \\
d & \delta
\end{array}\right)=\left(\begin{array}{l}
\alpha \gamma-(a, d), \alpha c+\delta a+b d \\
\gamma b+\beta d+\alpha c, \beta \delta-(b, c)
\end{array}\right) \text {. }
$$

Then $\mathfrak{A}$ is a flexible algebra over $\Phi$ in the sense that

$$
(x y) x=x(y x), x, y \in \mathfrak{A} \text {. }
$$

Furthermore $\mathfrak{A}$ is an alternative algebra over $\Phi$, i.e., $x^{2} y=x(x y)$ and $(y x) x=y x^{2}, x, y \in \mathfrak{X}$ if and only if the anti-commutative algebra $A$ has the following property:

$$
a(b c)=(a, c) b-(a, b) c, a, b, c \in A .
$$

This is checked easily by a comparison of entries of vector matrices $x^{2} y$ and $x(x y)$. We note that this property implies the Jacobi identity: $a(b c)+b(c a)+c(a b)=0$ and $A$ is a Lie algebra over the field $\Phi$.

We shall determine all the alternative algebras over $\Phi$ which are constructed from the Lie algebras with (2) by the Zorn vector matrices. First we determine all the Lie algebras with (2) and let $L$ be a finite 
dimensional Lie algebra over $\Phi$ equipped with an associative symmetric bilinear form $(a, b)$ and having the property (2). We return to writing $\left[\begin{array}{ll}a & b\end{array}\right]$ in place of $a b$. Set $L^{\perp}=\{a \in L \mid(a, b)=0, b \in L\}$ the radical of the bilinear form. If the bilinear form $(a, b)$ is nondegenerate, i.e., $L^{\perp}=0$, it follows from (2) that $L$ is a simple Lie algebra. On the other hand, if $(a, b)$ is degenerate we have the following.

LEMma. If the bilinear form $(a, b)$ is degenerate, then the Lie algebra $L$ is nilpotent with $L^{3}=0$ or $L=\Phi u+L^{\perp}$ where $L^{\perp}$ is a nonzero abelian ideal and $\left.(a d u)^{2}\right|_{L^{\perp}}=\rho I, \rho=-(u, u) \neq 0$ in $\Phi$.

Proof. If $L^{\perp}=L$, the condition (2) implies $L^{3}=0$. In the rest of the proof we assume that $L^{\perp} \neq L$, and $L^{\perp}$ is a nonzero proper ideal of $L$. There exists an element $u \neq 0$ in $L$ which is not in $L^{\perp}$ and satisfies $(u, u) \neq 0$. Let $\left(y_{1}, y_{2}, \cdots, y_{m}\right)$ be a basis for $L^{\perp}$.

$$
\left.(a d u)^{2}\right|_{L^{\perp}}=-(u, u) I
$$

because we have $(a d u)^{2} y_{i}=\left[u\left[u, y_{i}\right]\right]=-(u, u) y_{i}$ for all $y_{i}$. Since

$$
\rho=-(u, u) \neq 0
$$

in $\Phi$, the mapping ad $u$ is nonsingular on $L^{\perp}$.

$$
(a d u)\left[y_{i}, y_{j}\right]=\left(u, y_{j}\right) y_{i}-\left(u, y_{i}\right) y_{j}=0
$$

for all $i, j$ imply $\left[y_{i}, y_{j}\right]=0$ which means $L^{\perp}$ abelian. Finally we show that $L$ is the direct sum of two subspaces $\Phi u$ and $L^{\perp}$. Let $x$ be any element of $L$, not in $L^{\perp} . \quad(a d u)\left[x, y_{i}\right]=-(u, x) y_{i}$ and set $\tau=-(u, x)$. Then $\left.(a d u) a d(\tau u-\rho x)\right|_{L^{\perp}}=0$. Since $a d u$ is nonsingular on $L^{\perp}$, $\left.a d(\tau u-\rho x)\right|_{L^{\perp}}=0$. We wish to show that $(y, \tau u-\rho x)=0$ for any $y$ of $L$, which is equivalent to saying that $x \in \Phi u+L^{\perp}$. Since $\left[\tau u-\rho x, y_{i}\right]=0$ for all $y_{i}$ of the basis for $L^{\perp}, 0=\left[y\left[\tau u-\rho x, y_{i}\right]\right]=$ $-(y, \tau u-\rho x) y_{i}$. This has completed our proof.

Now we first take up the case the bilinear form $(a, b)$ on the Lie algebra $L$ is nondegenerate. It is known ([2]) that $(a, b)$ on $L$ is nondegenerate if and only if the algebra $\mathfrak{A}$ constructed from $L$ is simple. Since the alternative algebra $\mathfrak{A}$ is simple, $\mathfrak{A}$ is the split Cayley algebra or an associative algebra ([1]). We consider the latter case and follow Sagle's argument in [3]. Let

$$
x=\left(\begin{array}{ll}
\alpha & a \\
b & \beta
\end{array}\right), y=\left(\begin{array}{ll}
\gamma & c \\
d & \delta
\end{array}\right), z=\left(\begin{array}{ll}
\lambda & g \\
h & \mu
\end{array}\right)
$$

be any elements of $\mathfrak{A}$. By a comparison of $(1,1)$-entries of $(x y) z=$ 
$x(y z)$ we have $([b d], h)=(a,[c g])$. Without loss of generality we may take $a=0$ and we have $([b d], h)=0$ for all $h \in L$. It follows from the nondegeneracy that $[b d]=0$ for all $b, d$ of $L$, i.e., $L^{2}=0$. From $0=[a[b c]]=(a, c) b-(a, b) c$, we have $\operatorname{dim} L=1$ and therefore $\mathfrak{A}$ is a quaternion algebra. Hence we have the following

Theorem 1. Let $L$ be a finite dimensional Lie algebra over a field $\Phi$ of characteristic $\neq 2$ equipped with an associative symmetric bilinear form $(a, b)$ and having the property $(2)$. If $(a, b)$ is nondegenerate, then $\mathfrak{X}$ is the split Cayley algebra or a quaternion algebra.

A similar consideration to this theorem is given in [3]. As an immediate consequence of the theorem we have

COROLLARY. Let $L$ be as in Theorem 1. If the bilinear form $(a, b)$ is nondegenerate $L$ is simple with dimensionality three or one.

Next we consider the remaining case, that is, $(a, b)$ on $L$ is degenerate. Let $\left(u_{1}, u_{2}, \cdots, u_{n}\right)$ be a basis for $L$ over $\Phi$ and we set

$$
\begin{gathered}
e_{1}=\left(\begin{array}{ll}
1 & 0 \\
0 & 0
\end{array}\right), \quad e_{2}=\left(\begin{array}{ll}
0 & 0 \\
0 & 1
\end{array}\right), \\
e_{12}^{(s)}=\left(\begin{array}{cc}
0 & u_{s} \\
0 & 0
\end{array}\right), \quad e_{21}^{(s)}=\left(\begin{array}{ll}
0 & 0 \\
u_{s} & 0
\end{array}\right), \quad s=1,2, \cdots, n .
\end{gathered}
$$

These form a basis for the algebra $\mathfrak{A}$ over $\Phi$. Let $L=\Phi u+L^{\perp}$ be as in lemma and take the basis for $L$ to be $u_{1}=u$ and $\left(u_{2}, \cdots, u_{n}\right)$ a basis for the abelian ideal $L^{\perp}$. We have the following multiplication table for $\mathfrak{A}$ :

$$
\begin{aligned}
& e_{i} e_{j}=\delta_{i j} e_{i}, \\
& e_{i} e_{i k}^{(s)}=e_{i k}^{(s)} e_{k}=e_{i k}^{(s)}, \\
& e_{k} e_{i k}^{(s)} e_{i}=e_{i k}^{(s)} e_{i}=0, \\
& e_{i k}^{(s)} e_{k i}^{(t)}=\left\{\begin{array}{l}
\rho e_{i} \text { if }(s, t)=(1,1), \\
0 \text { otherwise, }
\end{array}\right. \\
& e_{i k}^{(s)} e_{i k}^{(t)}=-e_{i k}^{(t)} e_{i k}^{(s)}= \begin{cases}0 \text { if } s, t=2,3, \cdots, n, \\
x_{k i} \text { otherwise }\end{cases}
\end{aligned}
$$

where $i, j, k=1,2 ; i \neq k ; s, t=1,2, \cdots, n$ and $x_{k i}$ is a $2 \times 2$ vector matrix with 0 for all entries except for $(k, i)$-entry $\left[\begin{array}{ll}u_{s} & u_{t}\end{array}\right]$. The $e_{12}^{(s)}$ and $e_{21}^{(s)}, s=2,3, \cdots, n$ are all properly nilpotent and therefore generate the radical $\mathfrak{R}$ of $\mathfrak{A}$ (Zorn Theorem 3.7 in [4]). It follows that $\mathfrak{A}=$ $\mathfrak{S}+\mathfrak{R}$ (direct sum) where $\mathfrak{S}$ is a quaternion subalgebra with basis 
$\left(e_{1}, e_{2}, e_{12}^{(1)}, e_{21}^{(1)}\right)$. We note that this quaternion subalgebra $\subseteq$ is the same as one given in Theorem 1 . Now we consider the remaining case: $L^{\perp}=L$ and $L$ is nilpotent with $L^{3}=0$. Take a basis

$$
\left(u_{1}, \cdots, u_{m}, \cdots, u_{n}\right)
$$

for $L$ such that $\left(u_{m+1}, \cdots, u_{n}\right)$ is a basis for the abelian ideal $L^{2}$ of $L$. We have

$$
\begin{aligned}
& {\left[u_{i} u_{j}\right] \in L^{2}, 1 \leqq i, j \leqq m \text { and }} \\
& {\left[\begin{array}{ll}
u_{i} & u_{j}
\end{array}\right]=0 \text { otherwise. }}
\end{aligned}
$$

The multiplication table for $\mathfrak{A}$ is as follows:

$$
\begin{aligned}
& e_{i} e_{j}=\delta_{i j} e_{i}, \\
& e_{i} e_{i k}^{(s)}=e_{i k}^{(s)} e_{k}=e_{i k}^{(s)}, \\
& e_{k} e_{i k}^{(s)}=e_{i k}^{(s)} e_{i}=0, \\
& e_{i k}^{(s)} e_{k i}^{(t)}=-\left(u_{s}, u_{t}\right) e_{i}=0, \\
& e_{i k}^{(s)} e_{i k}^{(t)}=x_{k i}
\end{aligned}
$$

where $i, j, k=1,2 ; i \neq k ; s, t=1,2, \cdots, n$ and $x_{k i}$ is as before. The $e_{i k}^{(s)}, i \neq k, s=1,2, \cdots, n$ are all properly nilpotent and generate the radical $\mathfrak{R}$ of $\mathfrak{A}$. Hence $\mathfrak{A}$ is a direct sum of $\mathfrak{R}$ and a separable subalgebra $\Phi e_{1}+\Phi e_{2}$. We have proved the following

THEOREM 2. Let $L$ be as in Theorem 1. If the bilinear form $(a, b)$ is degenerate, then the algebra $\mathfrak{A}$ constructed from $L$ is a direct sum of its radical $\mathfrak{N}$ and a subalgebra $\mathfrak{\subseteq}$ where $\mathfrak{S}$ is either a quaternion or 2-dimensional separable algebra.

\section{BIBLIOGRAPHY}

1. E. Kleinfeld, Simple alternative rings, Ann. of Math. 58 (1953), 544-547.

2. L. J. Paige, $A$ note on noncommutative Jordan algebras, Portugaliae Math. 16 (1957), 15-18.

3. A. A. Sagle, Simple algebras that generalize the Jordan algebra $M_{3}{ }^{8}$, Canad. J. Math. 18 (1966) 282-290.

4. R. D. Schafer, An introduction to nonassociative algebras, New York, Academic Press, 1966.

5. M. Zorn, Theorie der alternativen Ringe, Abh. Math. Seit. Hamb. Univ. 8 (1930), 123-147.

Received November 1, 1968. This research was supported in part by the National Science Foundation under the grant AYE GY 3900.

East Tennessee State University

Johnson City, Tennessee 


\section{PACIFIC JOURNAL OF MATHEMATICS}

\section{EDITORS}

H. ROYDEN

Stanford University

Stanford, California

Richard Pierce

University of Washington

Seattle, Washington 98105
J. DugundJI

Department of Mathematics

University of Southern California

Los Angeles, California 90007

BASIL GORDON

University of California

Los Angeles, California 90024

\section{ASSOCIATE EDITORS}

E. F. BECKENBACH

B. H. NeUmanN

F. WOLF

K. YOSHIDA

\section{SUPPORTING INSTITUTIONS}

UNIVERSITY OF BRITISH COLUMBIA

CALIFORNIA INSTITUTE OF TECHNOLOGY

UNIVERSITY OF CALIFORNIA

MONTANA STATE UNIVERSITY

UNIVERSITY OF NEVADA

NEW MEXICO STATE UNIVERSITY

OREGON STATE UNIVERSITY

UNIVERSITY OF OREGON

OSAKA UNIVERSITY

UNIVERSITY OF SOUTHERN CALIFORNIA
STANFORD UNIVERSITY

UNIVERSITY OF TOKYO

UNIVERSITY OF UTAH

WASHINGTON STATE UNIVERSITY

UNIVERSITY OF WASHINGTON

$* * \quad * \quad *$
AMERICAN MATHEMATICAL SOCIETY
CHEVRON RESEARCH CORPORATION
TRW SYSTEMS
NAVAL WEAPONS CENTER




\section{Pacific Journal of Mathematics}

Vol. 30, No. $1 \quad$ September, 1969

William Wells Adams, Simultaneous diophantine approximations and cubic irrationals ..................................... 1

Heinz Bauer and Herbert Stanley Bear, Jr., The part metric in convex

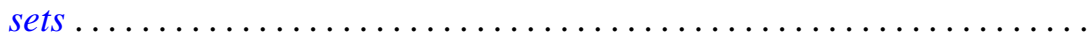

L. Carlitz, A note on exponential sums ...................... 35

Vasily Cateforis, On regular self-injective rings ................. 39

Franz Harpain and Maurice Sion, A representation theorem for measures on infinite dimensional spaces ......................... 47

Richard Earl Hodel, Sum theorems for topological spaces .............. 59

Carl Groos Jockusch, Jr. and Thomas Graham McLaughlin, Countable

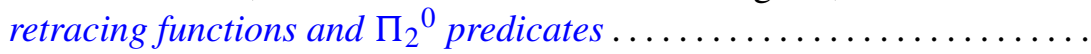

Bjarni Jónsson and George Stephen Monk, Representations of primary

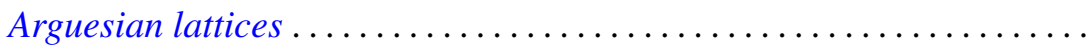

Virginia E. Walsh Knight, A continuous partial order for Peano continua...................................... 141

Kjeld Laursen, Ideal structure in generalized group algebras ........... 155

G. S. Monk, Desargues' law and the representation of primary lattices . . . 175

Hussain Sayid Nur, Singular perturbation of linear partial differential equation with constant coefficients ..........................

Richard Paul Osborne and J. L. Stern, Covering manifolds with cells ... 201

Keith Lowell Phillips and Mitchell Herbert Taibleson, Singular integrals in several variables over a local field...

James Reaves Smith, Local domains with topologically $T$-nilpotent radical....

Donald Platte Squier, Elliptic differential equations with discontinuous coefficients .................................

Tae-il Suh, Algebras formed by the Zorn vector matrix...

Earl J. Taft, Ideals in admissible algebras . .................... 259

Jun Tomiyama, On the tensor products of von Neumann algebras........ 263

David Bertram Wales, Uniqueness of the graph of a rank three group ..... 271

Charles Robert Warner and Robert James Whitley, A characterization of regular maximal ideals ......................... 